On a particular type of product manifolds and shear-free cosmological models

This article has been downloaded from IOPscience. Please scroll down to see the full text article.

2011 Class. Quantum Grav. 28175009

(http://iopscience.iop.org/0264-9381/28/17/175009)

View the table of contents for this issue, or go to the journal homepage for more

Download details:

IP Address: 139.179.139.67

The article was downloaded on 26/11/2012 at 15:56

Please note that terms and conditions apply. 


\title{
On a particular type of product manifolds and shear-free cosmological models
}

\author{
Metin Gürses $^{1}$, Matthias Plaue ${ }^{2}$ and Mike Scherfner ${ }^{2}$ \\ ${ }^{1}$ Department of Mathematics, Bilkent University, Ankara, Turkey \\ 2 Department of Mathematics, Technische Universität Berlin, Berlin, Germany \\ E-mail: gurses@fen.bilkent.edu.tr,plaue@math.tu-berlin.de and scherfner@math.tu-berlin.de
}

Received 28 March 2011, in final form 17 June 2011

Published 12 August 2011

Online at stacks.iop.org/CQG/28/175009

\begin{abstract}
Shear-free flows or observer fields are important objects of study in general relativity; stationary or rigid observers are important examples of shear-free reference frames. In this paper, we introduce a geometric structure based on a local coordinate expression of metrics admitting a shear-free reference frame. Furthermore, we investigate a large sub-class of these models ('tilted' warped products) that includes the Robertson-Walker spacetime, the Gödel spacetime and other models of Gödel type. We present a novel example of a rotating and expanding cosmological model that is contained in this class. Finally, we describe the geodesic barotropic perfect fluid solutions.
\end{abstract}

PACS numbers: $04.20 . \mathrm{Cv}, 04.20 . \mathrm{Jb}, 04.20 . \mathrm{Gz}$

\section{Introduction}

In general relativity, many known exact solutions of Einstein's equations admit symmetries such as being (conformally) stationary or static. The metric of spacetimes admitting such symmetries may locally be written in certain well-known standard forms, for example, a warped product in the static case. A considerable amount of work has been invested in finding out when such symmetries yield a global structure of this kind, a problem which is usually tied to issues of causality (cf for example [20]). In the following, we will derive and analyze a standard form for spacetimes admitting a shear-free reference frame, and apply the results in the construction of cosmological models.

Notation and conventions. Let $M$ be a $d$-dimensional smooth manifold, and $g$ a Riemannian or Lorentzian metric on $M$; the Lévi-Cività derivative associated with $g$ will be denoted by $\nabla$. We will also write $v \wedge w=v \otimes w-w \otimes v$ and $v \vee w=v \otimes w+w \otimes v$ for one-forms $v$ and $w$ on $M$. 
Now let $V$ be a reference frame, i.e. a cospacelike vector field $V$ on $M$ with $|g(V, V)|=1$. We will write $u:=g(V, \cdot)$ and $P:=g-\varepsilon u \otimes u$, where $\varepsilon=+1$ in the Riemannian and $\varepsilon=-1$ in the Lorentzian case.

The $(d-1)+1$-decomposition of the tensor field $(\nabla u)(X, Y):=g\left(\nabla_{X} V, Y\right)$ (with vector fields $X, Y)$ is then given by [19, p 83]

$$
\nabla u=\sigma-\omega+\frac{\Theta}{d-1} P+\varepsilon u \otimes \dot{u}
$$

with the kinematical quantities

$$
\begin{array}{ll}
\Theta=\operatorname{div} V & \begin{array}{l}
\text { expansion scalar, } \\
\text { acceleration, }
\end{array} \\
\dot{u}=g\left(\nabla_{V} V, \cdot\right) & \text { vorticity tensor, } \\
\omega=-\frac{1}{2}(\mathrm{~d} u+\varepsilon \dot{u} \wedge u) & \text { shear tensor. }
\end{array}
$$

Remark 1.1. Employing Einstein's summation convention, in local coordinates,

$$
\begin{aligned}
& \Theta=\nabla_{k} u^{k}, \quad \dot{u}_{i}=u^{k} \nabla_{k} u_{i}, \quad \omega_{i j}=-P_{i}^{k} P_{j}^{l} \nabla_{[k} u_{l]}, \\
& \sigma_{i j}=P_{i}^{k} P_{j}^{l} \nabla_{(k} u_{l)}-\frac{\Theta}{d-1} P_{i j} .
\end{aligned}
$$

Here, parentheses (brackets) denote (anti-)symmetrization. In this paper, we will use both index notation and coordinate-free notation.

We will mostly be concerned with the Lorentzian case and applications to general relativity. However, we will also treat the case $\varepsilon=+1$ in the proof of theorem 2.7.

\section{Shear-free reference frames and 'tilted' twisted products}

Shear-free reference frames frequently appear in general relativity. For example, any (conformally) stationary, Hubble-isotropic [18] or rigid reference frame is shear free. Furthermore, an important and still unsettled conjecture states that shear-free barotropic perfect fluids have vanishing vorticity or expansion [27]. Finally, it should be noted that a reference frame is shear free iff it is spatially conformally stationary [12, definition 3.1].

Locally, any metric admitting a shear-free reference frame can be brought into a standard form; see $[4,15]$.

Proposition 2.1. Let $(M, g)$ be a Riemannian or Lorentzian manifold admitting a shear-free reference frame $V, u=g(V, \cdot)$. Then about each point $p \in M$, there exists a chart $(U, \eta)$ with coordinates $\eta=\left(x^{0}, x^{1}, \ldots, x^{d-1}\right)=(t, x)$ such that $V=\partial_{t}$ and

$$
g_{i j}(t, x)=\varepsilon u_{i}(t, x) u_{j}(t, x)+a^{2}(t, x) h_{i j}(x),
$$

where $a$ is a function on $\eta(U)$.

Remark 2.2. Shear-free metrics with vanishing acceleration as well as vanishing expansion, i.e. with $a(t, x)=1$ and $\dot{u}_{i}(t, x)=0$, have been studied in [14, 16], called Gödel-type metrics.

Setting $b_{i}:=t_{, i}-\varepsilon u_{i}$ in the local expression (2.1) for shear-free manifolds, we see that the observer field is $g$-perpendicular to $b: u^{k} b_{k}=\delta^{k}{ }_{0} \delta^{0}{ }_{k}-\varepsilon \cdot u^{k} u_{k}=0$. This motivates the definition of the following geometric structure. 
Definition 2.3. Let $\varepsilon \in\{-1,+1\}$. We define the tilted twisted product of an interval $\left(I, \varepsilon \mathrm{d} t^{2}\right)$ with a connected Riemannian manifold $(N, h)$ to be the product manifold $M=I \times N$, furnished with the metric tensor

$$
g=\varepsilon\left(\pi_{1}^{*} \mathrm{~d} t-b\right)^{2}+a^{2} \pi_{2}^{*} h,
$$

where $a$ is a given real-valued, positive function on $M$ and $b$ is a one-form on $M$ which is tangent to each hypersurface $N_{s}:=\{s\} \times N$, i.e. $b\left(\partial_{t}\right)=0$, where $\partial_{t}$ is understood as the lift of the canonical vector field on $I$.

We will also write $(M, g)=\left(I, \varepsilon \mathrm{d} t^{2}\right) \times_{(b, a)}(N, h)$ for this structure. We have that $(M, g)$ is a connected Riemannian (Lorentzian) manifold for $\varepsilon=+1(\varepsilon=-1)$ with a shearfree reference frame $V=\partial_{t}$. Any metric admitting a shear-free reference frame is locally isometric to a tilted twisted product. If the twisting function $a$ depends only on $t$, we will speak of a tilted warped product. To keep the notation clear, we will omit the projections $\pi_{1}, \pi_{2}$ in the following. Of course, the terminology refers to twisted products [25] and tilted cosmological models [7].

Causal structure. In the following, let $\|\cdot\|$ be the norm induced by the Riemannian metric $h$, i.e. we have for example $\|b\|=\left(h^{-1}(b, b)\right)^{\frac{1}{2}}$, where $h^{-1}$ is understood as the lift of the inverse metric on $N$.

Proposition 2.4. A tilted twisted product spacetime $\left(I,-\mathrm{d} t^{2}\right) \times_{(b, a)}(N, h)$ is stably causal if $\|b\|<a$. It is non-chronological if $N$ is compact and there exists a slice $N_{s}$ with $\|b\|_{p}>a(p)$ for all $p \in N_{s}$.

Proof. It may be easily checked that the inverse of a tilted twisted product metric (2.2) is given by

$$
g^{-1}=\frac{1}{a^{2}}\left(h^{-1}+\left(\|b\|^{2}+\varepsilon a^{2}\right) \partial_{t} \otimes \partial_{t}+\partial_{t} \vee\left(h^{-1}(b, \cdot)\right)\right) .
$$

If $\|b\|<a$, it follows that the global coordinate $t$ is a temporal function [2]:

$$
g^{-1}(\mathrm{~d} t, \mathrm{~d} t)=\frac{\|b\|^{2}-a^{2}}{a^{2}}<0 .
$$

It is not difficult to see that in the Lorentzian case, at some point $p \in N_{s}$, the hyperplane tangent to $N_{s}$ is spacelike iff $\left\|b_{p}\right\|<a(p)$, timelike iff $\left\|b_{p}\right\|>a(p)$ and lightlike iff $\left\|b_{p}\right\|=a(p)$. If $\|b\|>a$ on a slice, it is a compact timelike hypersurface which must contain a closed timelike curve. (This may only happen if $N$ has vanishing Euler characteristic.)

Example 2.5. The non-chronological Gödel spacetime [13] with the metric

$$
g=-\mathrm{d} t^{2}-\mathrm{e}^{\sqrt{2} \omega_{0} x} \mathrm{~d} t \vee \mathrm{d} y+\mathrm{d} x^{2}-\frac{1}{2} \mathrm{e}^{2 \sqrt{2} \omega_{0} x} \mathrm{~d} y^{2}+\mathrm{d} z^{2}
$$

defined on $M=\mathbb{R} \times \mathbb{R}^{3}$ is a tilted product $(M, g)=\left(\mathbb{R},-\mathrm{d} t^{2}\right) \times_{(b, a)}\left(\mathbb{R}^{3}, h\right)$ with $h=\mathrm{d} x^{2}+\frac{1}{2} \mathrm{e}^{2 \sqrt{2} \omega_{0} x} \mathrm{~d} y^{2}+\mathrm{d} z^{2}$ and $b=-\mathrm{e}^{\sqrt{2} \omega_{0} x} \mathrm{~d} y$. It holds $\|b\|^{2}=2>1=a^{2}$.

Remark 2.6. Tilted twisted product spacetimes with $a=1$ and $\|b\|<1$ are conformally equivalent to standard stationary spacetimes, the causal structure of which has recently been studied in a number of articles [8, 11, 20]. 
Ricci curvature. In adapted coordinates $(t, x)=\left(t, x_{1}, \ldots, x_{d-1}\right)$, the expression for the inverse metric (2.3) reads

$$
g^{i j}=\frac{1}{a^{2}}\left(\bar{h}^{i j}+\left(\|b\|^{2}+\varepsilon a^{2}\right) u^{i} u^{j}+u^{i} \bar{b}^{j}+u^{j} \bar{b}^{i}\right),
$$

with $\bar{h}^{i k} h_{k j}=\delta^{i}{ }_{j}-\delta^{0}{ }_{j} \delta_{0}^{i}=\delta^{i}{ }_{j}-t_{j} u^{i}$ and $\bar{b}^{j}=\bar{h}^{j k} b_{k}=-\varepsilon \bar{h}^{j k} u_{k}$. Any time an index is raised with $\bar{h}^{i j}$, we will indicate this with an overbar.

The $(d-1)+1$-decomposition of the Ricci and scalar curvature of a tilted warped product (i.e. a tilted product metric (2.2) with a scale parameter that only depends on the coordinate $t$ ) is given as follows.

Theorem 2.7. $\quad$ Consider a tilted warped product $(M, g)=\left(I, \varepsilon \mathrm{d} t^{2}\right) \times_{(b, a)}(N, h)$ and introduce adapted coordinates $(t, x)$. Then, the components of the Ricci tensor of $(M, g)$ are given by

$$
\begin{aligned}
R_{i j}=r_{i j}-\dot{b}_{i} & \dot{b}_{j}-\mathrm{D}_{(i} \dot{b}_{j)}-\ddot{b}_{(i} b_{j)}-(d-3) \theta \mathrm{D}_{(i} b_{j)}-(d-5) \theta \dot{b}_{(i} b_{j)} \\
+ & \frac{2 \varepsilon}{a^{2}}\left[\bar{\omega}^{k}{ }_{j} \omega_{i k}+\left(2 \dot{\bar{b}}^{k}+(d-3) \theta \bar{b}^{k}\right) \omega_{k(j} u_{i)}+\bar{b}^{k} \dot{\omega}_{k(i} u_{j)}+u_{(j} J_{i)}\right] \\
+ & {\left[\frac{\|\omega\|^{2}}{a^{4}}-\frac{\varepsilon}{2 a^{2}}\left((d-3) \theta\left(\|b\|^{2}\right)^{\bullet}+\left(\|b\|^{2}\right)^{\bullet \bullet}+2 \mathrm{D}_{k} \dot{\bar{b}}^{k}\right)\right.} \\
& \left.-(d-1)\left(\theta^{2}+\dot{\theta}\right)\right] u_{i} u_{j} \\
+ & {\left[(d-3)\left(\theta^{2}-\dot{\theta}\right)\right] b_{i} b_{j}-2 \varepsilon(d-2) \dot{\theta} u_{(i} b_{j)}+2 \varepsilon \theta u_{(i} \dot{b}_{j)} } \\
+ & {\left[-\theta^{2}\left((d-3)\|b\|^{2}+\varepsilon(d-1) a^{2}\right)-\dot{\theta}\left(\|b\|^{2}+\varepsilon a^{2}\right)\right.} \\
& \left.-\theta\left(\left(\|b\|^{2}\right)^{\bullet}+\mathrm{D}_{k} \bar{b}^{k}\right)\right] h_{i j} .
\end{aligned}
$$

Here, $r_{i j}$ are the components of the Ricci tensor of $(N, h)$, and $\mathrm{D}$ is the Lévi-Cività derivative of the transversal metric $h$. (Both pulled back via the projection, i.e. $r_{i 0}=r_{0 i}=0$ and $\mathrm{D}_{0} b_{i}=0$ is understood.) Furthermore, $\theta:=(\log a)^{\bullet}=\frac{1}{3} \Theta$ is the Hubble parameter, $\omega_{i j}=-\varepsilon \mathrm{D}_{[i} b_{j]}$ the vorticity tensor and $J_{i}:=\mathrm{D}_{k} \bar{\omega}_{i}^{k}$. The scalar curvature $R$ of $(M, g)$ computes to:

$$
\begin{gathered}
a^{2} R=r-\varepsilon a^{-2}\|\omega\|^{2}-\left(\|b\|^{2}\right)^{\bullet \bullet}-(2 d-5) \theta\left(\|b\|^{2}\right)^{\bullet}-2 \mathrm{D}_{k} \dot{\bar{b}}^{k}-2(d-2) \theta \mathrm{D}_{k} \bar{b}^{k} \\
-\varepsilon(d-1) a^{2}\left(d \theta^{2}+2 \dot{\theta}\right)-(d-2)\|b\|^{2}\left((d-3) \theta^{2}+2 \dot{\theta}\right) .
\end{gathered}
$$

Proof. The proof consists of a series of straightforward but rather complicated computations. We will only note the most important steps; for detailed and extensive expositions on the $(3+1)$-formalism, we refer to the survey by Ellis and van Helst [10] and the book by Zelmanov [31].

If not otherwise noted, indices will be raised and lowered via the metric $g$, and partial derivatives will be denoted by commas. Note that the Christoffel symbols of the Lévi-Cività connection induced by the non-tilted, non-warped metric $\bar{g}=\varepsilon \mathrm{d} t^{2}+h$ may be written as

$$
\bar{\Gamma}_{i j}^{k}=\frac{1}{2} \bar{h}^{k n}\left(h_{n j, i}+h_{n i, j}-h_{i j, n}\right) \text {. }
$$

The Christoffel symbols $\Gamma_{i j}^{k}$ corresponding to $g$ are related to these via

$$
\Gamma_{i j}^{k}=\bar{\Gamma}_{i j}^{k}+\beta_{i j}^{k}+(\log a)^{\bullet} \Omega_{i j}^{k},
$$

where

$$
\begin{aligned}
& \beta^{k}{ }_{i j}:=\frac{\varepsilon}{2}\left(\left(u_{i \mid j}+u_{j \mid i}\right) u^{k}+u_{i} f_{j}^{k}+u_{j} f_{i}^{k}\right), \\
& \Omega^{k}{ }_{i j}:=a^{2} g^{k n}\left(t_{i} h_{n j}+t_{j} h_{n i}-t_{n} h_{i j}\right)
\end{aligned}
$$


with $f_{i j}:=u_{i, j}-u_{j, i}$, and denoting the Lévi-Cività derivative with respect to $\bar{g}$ by a vertical bar.

From the general formula for computing the Ricci tensor from the Christoffel symbols and by noting that $\beta^{k}{ }_{k j}=0$ and $\Omega^{k}{ }_{k j}=(d-1) t_{j}$, we conclude that

$$
\begin{gathered}
R_{i j}=\bar{R}_{i j}+\beta^{k}{ }_{i j \mid k}-\beta^{k}{ }_{i m} \beta^{m}{ }_{k j}+\theta^{2}\left((d-1) t_{k} \Omega_{i j}^{k}-\Omega_{i m}^{k} \Omega_{k j}^{m}\right)+\dot{\theta}\left(t_{k} \Omega^{k}{ }_{i j}-(d-1) t_{i} t_{j}\right) \\
+\theta\left(\Omega^{k}{ }_{i j \mid k}+(d-1) t_{k} \beta^{k}{ }_{i j}-\beta^{k}{ }_{i m} \Omega^{m}{ }_{k j}-\beta^{m}{ }_{k j} \Omega^{k}{ }_{i m}\right) .
\end{gathered}
$$

What now remains to do mostly involves the repeated application of the inverse metric formula (2.4) and the substitution $f_{i j}=2 \omega_{i j}+u_{i} \dot{b}_{j}-u_{j} \dot{b}_{i}$ with $\dot{b}_{i}:=u^{k} b_{i \mid k}=-\varepsilon \dot{u}_{i}$. For example, we have

$a^{2} f_{i k} f_{j}^{k}=4 \bar{h}^{k n} \omega_{n j} \omega_{i k}+2 \dot{\bar{b}}^{n}\left(\omega_{n j} u_{i}+\omega_{n i} u_{j}\right)-\|\dot{b}\|^{2} u_{i} u_{j}-\varepsilon a^{2} \dot{b}_{i} \dot{b}_{j}$,

$$
\begin{gathered}
a^{2} f_{i \mid k}^{k}=-4 \theta \bar{b}^{n} \omega_{n i}+2 \theta\left(\bar{b}^{n} \dot{b}_{n}\right) u_{i}+2 \bar{h}^{k n} \omega_{n i \mid k}+2 \bar{b}^{n} \dot{\omega}_{n i}+2 \dot{\bar{b}}^{n} \omega_{n i}+a^{2} \ddot{b}_{i}-\left(\bar{b}^{n} \dot{b}_{n}\right)^{\bullet} u_{i} \\
+\varepsilon\left(\bar{b}^{n} \dot{b}_{n}\right) \dot{b}_{i}-\left(\dot{\bar{b}}_{\mid k}^{k}\right) u_{i}+\varepsilon \dot{\bar{b}}^{k} b_{i \mid k}
\end{gathered}
$$

and $f_{m n} f^{m n}=\frac{4}{a^{4}}\|\omega\|^{2}+\frac{2 \varepsilon}{a^{2}}\|\dot{b}\|^{2}$ with $\|\omega\|^{2}:=\bar{h}^{i m} \bar{h}^{j n} \omega_{i j} \omega_{m n}$. Computing the other terms in a similar manner, plugging them into (2.5) and noting that $\bar{R}_{i j}=r_{i j}$ and $b_{i \mid j}=D_{j} b_{i}+\dot{b}_{i} t_{j}$ yield the final expression for the Ricci tensor. The scalar curvature of course follows from computing the trace (with respect to $g$ ).

For the remainder of this work, we will be concerned with applications in general relativity, and the following will be useful.

Corollary 2.8. Suppose Einstein's equations $R_{i j}-\frac{R}{2} g_{i j}=\tau_{i j}$ hold for a tilted warped product spacetime $\left(I,-\mathrm{d} t^{2}\right) \times_{(b, a)}(N, h)$ with $d=4$. Let the usual $(3+1)$-decomposition of the energy-momentum tensor be given by

$$
\tau_{i j}=\rho u_{i} u_{j}+p^{*} P_{i j}+2 q_{(i} u_{j)}+\Pi_{i j},
$$

with energy density $\rho$, (effective) pressure $p^{*}$, heat flux $q_{i}$ and anisotropic pressure $\Pi_{i j}$. Then,

$$
\begin{aligned}
& a^{2} \rho=\frac{r}{2}+\frac{3\|\omega\|^{2}}{2 a^{2}}-\theta\left(\|b\|^{2}\right)^{\bullet}-2 \theta \mathrm{D}_{k} \bar{b}^{k}+3 a^{2} \theta^{2}-\|b\|^{2}\left(\theta^{2}+2 \dot{\theta}\right), \\
& \begin{array}{r}
3 a^{2} p^{*}=-\frac{r}{2}+\frac{\|\omega\|^{2}}{2 a^{2}}+2 \theta\left(\|b\|^{2}\right)^{\bullet}+\left(\|b\|^{2}\right)^{\bullet \bullet}+2 \mathrm{D}_{k} \dot{\bar{b}}^{k}+2 \theta D_{k} \bar{b}^{k}-9 a^{2} \theta^{2}-6 a^{2} \dot{\theta} \\
\quad+\|b\|^{2}\left(\theta^{2}+2 \dot{\theta}\right),
\end{array} \\
& \begin{array}{r}
q_{i}=-\frac{2}{a^{2}}\left(\left(2 \dot{\bar{b}}^{k}+\theta \bar{b}^{k}\right) \omega_{k i}+\bar{b}^{k} \dot{\omega}_{k i}+J_{i}\right)+4 \dot{\theta} b_{i}-2 \theta \dot{b}_{i}, \\
\Pi_{i j}=r_{i j}-\dot{b}_{i} \dot{b}_{j}-\mathrm{D}_{(i} \dot{b}_{j)}-\ddot{b}_{(i} b_{j)}-\theta \mathrm{D}_{(i} b_{j)}+\theta \dot{b}_{(i} b_{j)}-\frac{2}{a^{2}} \bar{\omega}_{j}^{k} \omega_{i k}+\left(\theta^{2}-\dot{\theta}\right) b_{i} b_{j}-\alpha h_{i j},
\end{array}
\end{aligned}
$$

where the function $\alpha$ is determined by the condition $\bar{h}^{i j} \Pi_{i j}=0$.

Proof. This is a straightforward computation using the formulas

$$
\begin{aligned}
& \rho=R_{k l} u^{k} u^{l}+\frac{R}{2}, \quad 3 p^{*}=R_{k l} u^{k} u^{l}-\frac{R}{2}, \\
& q_{i}=a^{2} h_{i}^{k} R_{k l} u^{l}, \quad \Pi_{i j}=a^{4} h_{i}^{k} h_{j}^{l} R_{k l}-(\cdots) h_{i j} .
\end{aligned}
$$




\section{The tilted closed Robertson-Walker model}

As an application of tilted warped products and the Ricci and scalar curvature formulas stated in theorem 2.7, in this section we will provide an explicit example of a conformally stationary tilted warped product spacetime. For another example of a closed rotating and expanding world model, see [5].

First, we note the following general fact.

Proposition 3.1. The standard reference frame $V=\partial_{t}$ of a tilted warped product is parallel to a local conformal vector field iff the shift is of the form

$$
b_{j}(t, x)=a(t) \cdot\left(\chi_{j}(x)+\int_{0}^{t} \frac{\mathrm{d} \tau}{a(\tau)} \cdot \xi_{j}(x)\right),
$$

where $\xi_{j}(x)$ are the components of a closed one-form defined on the fiber $N: \mathrm{D}_{[i} \xi_{j]}=0$.

Proof. It is well known that a shear-free reference frame $V$ with $u=g(V, \cdot)$ is parallel to a local conformal vector field iff the one-form $\dot{u}-\theta u$ is closed; cf for example [17]. For tilted product spacetimes, this translates to the set of equations

$$
\ddot{b}_{j}=\left(\theta b_{j}\right)^{\bullet}, \quad \mathrm{D}_{[i} \dot{b}_{j]}=\theta \mathrm{D}_{[i} b_{j]} .
$$

The first equation may be readily integrated to give the desired expression for $b_{j}(t, x)$, whereas the second yields the constraint for $\xi_{j}(x)$.

The proposition holds globally if the fiber $N$ and therefore $M=I \times N$ is simply connected. Thus, the examples of tilted warped product spacetimes with a conformally stationary reference frame may be easily constructed. Such examples are of importance since these models are parallax free [17]. One such example is given in the following. Set up the coordinates $(r, \mu, v)$ on $S^{3} \subset \mathbb{R}^{4}$ defining the parametrization [30]:

$$
\begin{aligned}
& {[0,1] \times[0,2 \pi]^{2} \rightarrow S^{3},} \\
& (r, \mu, v) \mapsto\left(r \cos \mu, r \sin \mu, \sqrt{1-r^{2}} \cos v, \sqrt{1-r^{2}} \sin v\right) .
\end{aligned}
$$

Now consider the manifold $M=I \times S^{3}$ with a suitable interval $I \subset \mathbb{R}$, equipped with the tilted warped product metric

$g=-\left(\mathrm{d} t-b_{0} a\left(r^{2} \mathrm{~d} \mu+\left(1-r^{2}\right) \mathrm{d} \nu\right)^{2}+a^{2}\left(\frac{1}{1-r^{2}} \mathrm{~d} r^{2}+r^{2} \mathrm{~d} \mu^{2}+\left(1-r^{2}\right) \mathrm{d} \nu^{2}\right)\right.$,

where $b_{0} \in \mathbb{R}$ and $a$ is a function defined on $I$. The spatial metric $h$ is just the canonical metric on $S^{3}$. Provided that $b_{0} \neq 0$, the shift one-form $b$ does not vanish anywhere and is in fact, for each fixed $s \in I, h$-metrically equivalent to the left-invariant vector field on $S^{3}$, $\bar{b}=b_{0} a(s)\left(\partial_{\mu}+\partial_{\nu}\right)$.

The spacetime $(M, g)$ generalizes the closed Robertson-Walker spacetime. We have $\|b\|^{2}=b_{0}^{2} a^{2}$, and $(M, g)$ is stably causal for $\left|b_{0}\right|<1$ by virtue of proposition 2.4. Furthermore, the fiber $S^{3}$ is compact, and the spacetime is thus totally vicious for $\left|b_{0}\right|>1$ (i.e. contains a closed timelike curve through every point) since the observer field $V=\partial_{t}$ is conformally stationary by proposition 3.1 (cf [21, p 113]).

We will follow the rationale in [6] and assume the energy-momentum tensor to be of a viscous fluid form:

$$
\tau=\rho u \otimes u+(p-\zeta \Theta) P+q \vee u-2 \eta \sigma
$$

where $\zeta, \eta \geqslant 0$ are the functions representing the bulk and shear viscosity coefficients, respectively. 
Since the model is shear free, the form of the energy-momentum tensor (3.1) enforces that the anisotropic pressure vanishes. Since $h$ is an Einstein metric (i.e. $r_{i j}=\frac{r}{3} h_{i j}$ ) and $\bar{b}$ is a Killing vector field on the slices (i.e. $\left.\mathrm{D}_{(i} b_{j}\right)=0$ ), it may be readily verified via corollary 2.8 that this condition is equivalent to

$$
b_{i} b_{j}\left(-\ddot{a} a+(\dot{a})^{2}\right)-\bar{\omega}_{j}^{k} \omega_{i k}=\frac{1}{3}\left(\|b\|^{2}\left(-\ddot{a} a+(\dot{a})^{2}\right)+\|\omega\|^{2}\right) h_{i j} .
$$

Noting that $\omega_{i j}=\mathrm{D}_{[i} b_{j]}$, this equation may be evaluated in a straightforward manner. One then sees that in the case $b_{0} \neq 0$, the anisotropic pressure vanishes iff the scale factor satisfies the differential equation

$$
\ddot{a} a-(\dot{a})^{2}+1=0 .
$$

Note that the standard Robertson-Walker model (i.e. the case $b_{0}=0$ ) is shear free for any scale parameter. Introducing vorticity in the way as done here thus yields a tight constraint that seriously limits cosmic evolution and matter models.

Using this equation to eliminate the derivatives of $a$ of order larger than 1, the remaining components of the energy-momentum tensor are as follows:

$$
\begin{aligned}
& \rho=a^{-2}\left(-3(\dot{a})^{2}\left(b_{0}^{2}-1\right)+5 b_{0}^{2}+3\right), \\
& p-\zeta \Theta=a^{-2}\left(3(\dot{a})^{2}\left(b_{0}^{2}-1\right)-b_{0}^{2}+1\right), \\
& q=-4 a^{-2} b .
\end{aligned}
$$

Note in particular that in the case of a vanishing bulk viscosity coefficient, $\zeta=0$, the fluid is barotopic with the equation of state

$$
\rho=-p+4 a^{-2}\left(b_{0}^{2}+1\right) \text {. }
$$

We would also like to note that the model is purely Weyl electric.

As done in [6], we will in the following stipulate the thermodynamical conditions:

$$
\operatorname{div}(n \cdot V)=0, \quad \text { particle number conservation law, }
$$

$$
\begin{aligned}
& \mathrm{d}\left(\frac{S}{n}\right)=\frac{1}{T} \mathrm{~d}\left(\frac{\rho}{n}\right)+\frac{p}{T} \mathrm{~d}\left(\frac{1}{n}\right), \quad \text { Gibbs' relation, } \\
& q=-k P\left(\nabla T+T \nabla_{V} V, \cdot\right), \quad \text { temperature gradient law, }
\end{aligned}
$$

where $n, S, k, T \geqslant 0$ are the particle number density, entropy density, thermal conductivity and temperature, respectively. These conditions are designed so that the second law of thermodynamics, $\operatorname{div}\left(S V+\frac{1}{T} q^{\sharp}\right) \geqslant 0$, holds. Note, however, that more recent treatments of relativistic thermodynamics suggest that these constraints are too simple to give a full account of the actual physics. For an overview of different approaches of the problem, cf for example [1]. Like in the non-tilted case, particle conservation (3.3) enforces $n \propto a^{-3}$.

Vanishing bulk viscosity. For simplicity and to provide a more concrete example, we will also make the following additional assumptions.

(1) Each of the thermodynamical variables only depends on the 'cosmological development parameter' $t$ (remember that $t$ is only a temporal function for $\left|b_{0}\right|<1$ );

(2) bulk viscosity effects are negligible, i.e. $\zeta=0$;

(3) the entropy density satisfies the equation of state $S=\frac{\rho+p}{T}$ (cf [1, section 14.1] with vanishing diffusion). 
Accounting for (3.2), we may solve (3.5) for the thermal conductivity: $k=\frac{4}{a(a T)^{\bullet}}$.

With the above constraints, Gibbs' relation (3.4) also simplifies considerably and may be solved with respect to the temperature ( $T_{0}$ is some positive constant):

$$
T=T_{0} \cdot a^{-\frac{b_{0}^{2}-1}{b_{0}^{2}+1}}
$$

We may now also state explicit formulas for the entropy density and the thermal conductivity:

$$
S=\frac{4}{T_{0}}\left(b_{0}^{2}+1\right) a^{-\frac{b_{0}^{2}+3}{b_{0}^{2}+1}}, \quad k=\frac{2}{T_{0} \dot{a}}\left(b_{0}^{2}+1\right) a^{-\frac{2}{b_{0}^{2}+1}} .
$$

In order for the thermal conductivity to be well defined and non-negative, the expansion must be positive: $\dot{a}>0$. The entropy production is given by

$$
\operatorname{div}\left(S V+\frac{1}{T} q^{\sharp}\right)=\frac{8 b_{0}^{2}}{T_{0}\left(b_{0}^{2}+1\right)} \dot{a} a^{-\frac{2\left(b_{0}^{2}+2\right)}{b_{0}^{2}+1}} .
$$

We have yet to determine the scale factor. The isotropic-pressure condition (3.2) may actually be solved analytically via the substitution $a=\mathrm{e}^{f}$, yielding the autonomous equation $\ddot{f}+\mathrm{e}^{-2 f}=0$, the integration of which is elementary. The solutions are

$$
\begin{array}{ll}
a_{-}(t)=\alpha \sinh \left(\frac{t-t_{0}}{\alpha}\right), & t_{0}<t, \\
a_{0}(t)=t-t_{0}, & t_{0}<t, \\
a_{+}(t)=\frac{1}{\omega} \sin \left(\omega\left(t-t_{0}\right)\right), & t_{0}<t<\frac{\pi}{\omega}+t_{0} .
\end{array}
$$

In any case, the spacetime features a singularity. These three classes of solutions may be characterized by the deceleration parameter, $-\frac{a \ddot{a}}{\dot{a}^{2}}$, which is non-positive (zero, non-negative) for $a_{-}\left(a_{0}, a_{+}\right)$. Note that $a_{+}$violates the condition $\dot{a}>0$ and $k(t)$ becomes singular as $t$ approaches $t_{0}+\frac{\pi}{2 \omega}$. Also note that $\lim _{\alpha \rightarrow \infty} a_{-}(t)=\lim _{\omega \rightarrow 0} a_{+}(t)=a_{0}(t)$.

With the help of corollary 1 in [22], it is easy to check that the dominant and strong energy conditions are satisfied for $a=a_{0}$ and $a=a_{+}$. In the case $a=a_{-}$, the strong energy condition is satisfied iff $\left|b_{0}\right| \geqslant 1$ and the dominant and weak energy conditions are satisfied iff $\left|b_{0}\right| \leqslant 1$.

Dust solutions. We will now drop the constraint $\zeta=0$ and instead have a look at the viscous dust solutions of the model, i.e. $p=0$. This condition implies

$$
\zeta=\frac{1-b_{0}^{2}}{3 a \dot{a}}\left(3(\dot{a})^{2}-1\right)
$$

In order for $\zeta$ to be well defined and non-negative, we must have $a=a_{0}$ or $a=a_{-}$, and $\left|b_{0}\right| \leqslant 1$. Furthermore, the temperature is constant $\left(T=T_{0}\right)$, and the other thermodynamical variables compute to

$$
S=\frac{1}{a^{2} T_{0}}\left(3\left(1-b_{0}^{2}\right)(\dot{a})^{2}+3 b_{0}^{2}+5\right), \quad k=\frac{4}{T_{0} a \dot{a}} .
$$

Entropy production is given by

$$
\operatorname{div}\left(S V+\frac{1}{T} q^{\sharp}\right)=\frac{\dot{a}}{a^{3} T_{0}}\left(9\left(1-b_{0}^{2}\right)(\dot{a})^{2}+b_{0}^{2}+3\right) .
$$


Geodesics and observations. We wish to show some physical features of the (expanding) tilted Robertson-Walker universe a fluid observer traveling along an integral curve of $V=\partial_{t}$ would experience. First, an orthonormal tetrad is given by

$$
\begin{aligned}
& \eta_{0}=\partial_{t}, \quad \eta_{1}=b_{0} \partial_{t}+a^{-1}\left(\partial_{\mu}+\partial_{\nu}\right), \\
& \eta_{2}=\frac{1}{a r \sqrt{1-r^{2}}}\left(-\left(1-r^{2}\right) \partial_{\mu}+r^{2} \partial_{\nu}\right), \quad \eta_{3}=\frac{\sqrt{1-r^{2}}}{a} \partial_{r} .
\end{aligned}
$$

Note that $\eta_{1}$ in fact points in the direction of the fluid's acceleration and along its rotational axis. The integral curves of the vector fields

$$
\xi_{ \pm}=a^{-1}\left(\eta_{0} \pm \eta_{1}\right)=a^{-1}\left(\left(1 \pm b_{0}\right) \partial_{t} \pm a^{-1}\left(\partial_{\mu}+\partial_{\nu}\right)\right)
$$

are null geodesics, i.e. paths of light rays. The integral curves of $\partial_{\mu}+\partial_{v}$ may be visualized as closed curves running around the two-dimensional tori embedded in $S^{3}$, given by $r=$ const.

Without loss of generality, we will assume $t_{0}=0$ and $b_{0} \geqslant 0$. For the case $a=a_{0}$, the integral curves of $\xi_{ \pm}$are given by

$$
\begin{aligned}
& t(\tau)=\sqrt{2\left(1 \pm b_{0}\right) \tau+t(0)^{2}}, \quad r(\tau)=\mathrm{const}, \\
& \mu(\tau(t))=v(\tau(t))= \pm\left(1 \pm b_{0}\right)^{-1} \log \left(\frac{t(\tau)}{t(0)}\right),
\end{aligned}
$$

provided that $b_{0} \neq 1$. Here, we assumed $\mu(0)=v(0)=0$. If $b_{0}=1$, the null geodesics determined by $\xi_{-}$are closed for any scale parameter $a(t)$. This implies that a hypothetical human observer traveling along the fluid flow and constantly looking along the direction $\mp \eta_{1}$ would, at any time $t=t_{2}$, see an image of the back of his head from the point in time $t_{1}=\mathrm{e}^{-2 \pi\left(1 \pm b_{0}\right)} t_{2}$. Note that the causality violation of this spacetime for the case $b_{0}>1$ becomes physically manifest as the observer would see a future image of himself in the direction of $+\eta_{1}$. This yields the usual causal paradoxes that would occur if, for example, the observer decided to change the outcome of his observation.

In the case $a=a_{-}$, the time it takes for the light rays emitted by the observer to return is determined by

$$
\tanh \left(\frac{t_{1}}{2 \alpha}\right)=\mathrm{e}^{-2 \pi\left(1 \pm b_{0}\right)} \tanh \left(\frac{t_{2}}{2 \alpha}\right)
$$

Thus, the light from the observer in the direction of $+\eta_{1}$ only returns if it was emitted during the epoch $t<\alpha \log \left(\operatorname{coth}\left(\pi\left(1+b_{0}\right)\right)\right)$. The light emitted in the $-\eta_{1}$ direction meets the observer again if it was emitted at times $t<\alpha \log \left(\operatorname{coth}\left(\pi\left(1-b_{0}\right)\right)\right)$ provided that $b_{0}<1$-it always returns if $b_{0}>1$, again from a future event on the observer's worldline.

As for examples of timelike geodesics, in the case $b_{0}<1$ the observer field $W=$ $\cosh (\phi(t)) \eta_{0}-\sinh (\phi(t)) \eta_{1}$ with

$$
\tanh (\phi(t))=\frac{c b_{0} a(t)^{2} \pm\left(1-b_{0}^{2}\right) \sqrt{1+c a(t)^{2}}}{c a(t)^{2}+1-b_{0}^{2}}
$$

or $\tanh (\phi(t))=b_{0}$ has vanishing acceleration. For any $v_{0} \neq b_{0}$ with $-1<v_{0}<1$ and $t_{1}>0$, we may choose a sign and the constant $c>0$ such that $\tanh \phi\left(t_{1}\right)=v_{0}$. Observe that we have $\lim _{t \rightarrow \infty} \tanh \phi(t)=b_{0}$. Thus, a freely falling test particle emitted by the observer in the direction of $-\eta_{1}$ with the arbitrary Newtonian velocity $v=v_{0}$ will accelerate with respect to the fluid in this (or the opposite) direction in order to eventually reach the constant terminal velocity $v=b_{0}$. Similar to light signals, the test particle may return to the observer or arbitrarily close. 


\section{Barotropic perfect fluid and dust solutions with geodesic flow}

We now wish to classify tilted warped product spacetimes with a geodesic perfect fluid source, i.e. we have $\dot{b}_{i}=0$ and

$$
R_{i j}-\frac{R}{2} g_{i j}=(\rho+p) u_{i} u_{j}+p g_{i j}
$$

where $\rho+p \neq 0$ on any open subset. Furthermore, we require the fluid to be barotropic, i.e. an equation of state $\rho=\rho(p)$ with $\frac{\mathrm{d} \rho}{\mathrm{d} p} \neq 0$ holds. Kinematically, models with vanishing acceleration and shear are important since they follow an isotropic Hubble law of first order [18].

Proposition 4.1. For any (four-dimensional) simply-connected tilted warped product spacetime $(M, g)$ with a barotropic geodesic perfect fluid source (and fluid vector field $\left.V=\partial_{t}\right)$, either one of the following two cases may occur.

(1) The vorticity vanishes. In this case, $(M, g)$ is isometric to a Robertson-Walker spacetime. Furthermore, $(N, h)$ is conformally equivalent to a space of constant curvature.

(2) The expansion vanishes. In this case, the following hold.

(a) The pressure $p$, energy density $\rho$ and rotation scalar $\|\omega\|^{2}$ are constant.

(b) The fiber $(N, h)$ is a space of constant scalar curvature.

(c) The fiber $(N, h)$ is a space of constant curvature iff the vorticity also vanishes. In this case, $(M, g)$ is a static Robertson-Walker spacetime. It is Einstein's universe provided that $\rho>0$ holds and $(N, h)$ is complete.

Proof. In the following, we will make frequent use of corollary 2.8. By a RobertsonWalker spacetime, we mean a warped product $\left(\tilde{I} \times N,-\mathrm{d} t^{2}+\tilde{h}\right)$ with a fiber $(N, \tilde{h})$ of constant curvature (which in $d-1=3$ dimensions is equivalent to $(N, \tilde{h})$ being Einstein [3, proposition 1.120]). One may also require the fiber to be complete in which case the spacetime is not only stably causal but globally hyperbolic [26]. Since the shear-free fluid conjecture is known to be true for vanishing acceleration, either the vorticity or the expansion vanishes [27]. Let us first examine the case of vanishing vorticity. In this case, we must have $b=\mathrm{d} f$ with some function $f: N \rightarrow \mathbb{R}$ (since we assume $N$ to be simply connected, [24, pp 358-60]). Furthermore, the heat flux vanishes iff $\dot{\theta}=0$ or $b_{i}=0$. If the shift vanishes, $b_{i}=0$, we are finished since the vanishing of the anisotropic pressure immediately yields $r_{i j}=\alpha h_{i j}$. Thus, $a(t)=c \mathrm{e}^{\theta t}$ for some positive constant $c$. If we pull back the metric

$$
g=-(\mathrm{d} t-\mathrm{d} f)^{2}+c^{2} \mathrm{e}^{2 \theta t} h
$$

via the diffeomorphism $\Phi(t, x)=(t+f(x), x)$, we see that it becomes a warped product:

$$
\Phi^{*} g=-\mathrm{d} t^{2}+c^{2} \mathrm{e}^{2 \theta t} \mathrm{e}^{2 \theta f} h .
$$

The fiber metric $\tilde{h}=\mathrm{e}^{2 \theta f} h$ is conformally equivalent to $h$; the Ricci tensor may be computed with the usual formula [3, theorem 1.159]

$$
\begin{aligned}
\tilde{r}_{i j} & =r_{i j}-\theta \mathrm{D}_{i} f_{, j}+\theta^{2} f_{, i} f_{, j}-(\cdots) h_{i j} \\
& =r_{i j}-\theta \mathrm{d}_{(i} b_{j}+\theta^{2} b_{i} b_{j}-(\cdots) h_{i j} .
\end{aligned}
$$

Plugging this into the formula for the anisotropic pressure, we have $0=\Pi_{i j}=\tilde{r}_{i j}-\tilde{\alpha} \tilde{h}_{i j}$ as desired.

For the second case of vanishing expansion ( $a=$ const), the conditions of a vanishing heat flux and anisotropic pressure yield

$$
\bar{\rho}=\frac{1}{2} r+\frac{3}{2}\|\bar{\omega}\|^{2}, \quad \bar{p}=-\frac{1}{6} r+\frac{1}{6}\|\bar{\omega}\|^{2}, \quad \mathrm{D}_{k} \bar{\omega}_{i}^{k}=0,
$$




$$
r_{i j}=2 \bar{\omega}_{i}^{k} \bar{\omega}_{k j}+\frac{1}{3}\left(2\|\bar{\omega}\|^{2}+r\right) h_{i j}
$$

where $\bar{\rho}=a^{2} \rho, \bar{p}=a^{2} p$ and $\bar{\omega}_{i j}=a \omega_{i j}$. It is clear from these formulas and momentum conservation $\left(h_{j}^{m} p_{, m}=-a^{2}(\rho+p) \dot{b}_{j}=0\right)$ that the gradient of $p$ vanishes. The equation of state then implies that $\rho$ and therefore $r$ and $\|\omega\|^{2}$ are constant, too.

For the last claim (c), observe that equation (4.4) in an orthonormal basis shows that the 3-geometry satisfies the Einstein condition, i.e. has constant curvature, iff the antisymmetric matrix $\left(\bar{\omega}_{i j}\right)$ vanishes or is a non-trivial square root of a multiple of the identity matrix; but the latter is not possible in three dimensions. The spacetime is thus a static Robertson-Walker solution.

Since shear-free dusts also either expand or rotate [9], we may state by very similar arguments that:

Proposition 4.2. For any simply connected tilted warped product spacetime $(M, g)$ with a geodesic dust source, either one of the two following cases may occur.

(1) The vorticity vanishes. In this case, $(M, g)$ is a Robertson-Walker dust solution.

(2) The expansion vanishes. In this case, the vorticity also vanishes iff the spacetime is flat.

Example 4.3. The Gödel spacetime is an example of a rotating spacetime covered by proposition 4.1; the van Stockum spacetime $[23,28]$ is a rotating dust solution covered by proposition 4.2 .

\section{Summary and outlook}

Apart from constructing further explicit examples of shear-free cosmological models and investigating other more general classes (for example, rigidly rotating matter), many interesting subjects remain, among which are the following.

- The causal structure of tilted product spacetimes with $\|b\|=a$, or with $\|b\|>a$ and non-compact fiber (like the classical Gödel spacetime).

- The curvature structure of the general twisting case $a=a(t, x)$.

- Necessary and sufficient conditions for a shear-free spacetime to be globally of a tilted product type, or conformally equivalent to a tilted product.

Finally, one might also want to investigate the Riemannian case, for which we also expect interesting applications. For example, shear-free reference frames with vanishing expansion define Riemannian flows [29] with a normal bundle metric $P$.

\section{Acknowledgments}

MG is partially supported by the Scientific and Technological Research Council of Turkey (TÜBITAK) and Turkish Academy of Sciences (TÜBA). We would also like to thank the referees for their helpful comments and suggestions.

\section{References}

[1] Andersson N and Comer G L 2007 Relativistic fluid dynamics: physics for many different scales Living Rev. Rel. 101 (http://www.livingreviews.org/lrr-2007-1)

[2] Bernal A N and Sánchez M 2005 Smoothness of time functions and the metric splitting of globally hyperbolic spacetimes Commun. Math. Phys. 257 43-50 
[3] Besse A 1987 Einstein Manifolds (Berlin: Springer)

[4] Chrobok T, Obukhov Y N and Scherfner M 2001 On the construction of shear-free cosmological models Mod. Phys. Lett. A $201321-5$

[5] Chrobok T, Obukhov Y N and Scherfner M 2002 Shear-free rotating inflation Phys. Rev. D 66043518

[6] Coley A A 1989 On the thermodynamics of cosmological models with heat conduction Phys. Lett. A 137 235-8

[7] Coley A A, Hervik S and Lim W C 2006 Fluid observers and tilting cosmology Class. Quantum Grav. 23 3573-91

[8] Dirmeier A, Plaue M and Scherfner M Growth conditions, Riemannian completeness and Lorentzian causality J. Geom. Phys. (at press) doi:10.1016/j.geomphys.2011.04.017

[9] Ellis G F R 1967 Dynamics of pressure-free matter in general relativity J. Math. Phys. 29 1171-94

[10] Ellis G F R and van Elst H 1999 Cosmological models (Cargèse lectures 1998) Theoretical and Observational Cosmology (NATO Adv. Study Inst. Ser. C. Math. Phys. Sci. vol 541) ed M Lachièze-Rey (Kluwer) pp 1-113 (arXiv:gr-qc/9812046)

[11] Flores J L 2009 The causal boundary of standard stationary spacetimes AIP Conf. Proc. 1122 272-5

[12] García-Río E and Kupeli D N 1996 Singularity versus splitting theorems for stably causal spacetimes Ann. Glob. Anal. Geom. 14 301-12

[13] Gödel K 1949 An example of a new type of cosmological solutions of Einstein's field equations of gravitation Rev. Mod. Phys. 21 447-50

[14] Gürses M, Karasu A and Sarioglu O 2005 Gödel-type metrics in various dimensions Class. Quantum Grav. 22 1527-43

[15] Gürses M, Plaue M, Scherfner M, Schönfeld T and de Sousa L A M Jr 2010 On spacetimes with given kinematical invariants: construction and examples Gödel-type Spacetimes: History and New Developments (Vienna: Kurt Gödel Society)

[16] Gürses M and Sarioglu O 2005 Gödel-type metrics in various dimensions: II. Inclusion of a dilaton field Class. Quantum Grav. 22 4699-713

[17] Hasse W and Perlick V 1988 Geometrical and kinematical characterization of parallax-free world models J. Math. Phys. 29 2064-8

[18] Hasse W and Perlick V 1999 On spacetime models with an isotropic Hubble law Class. Quantum Grav. 16 2559-76

[19] Hawking S W and Ellis G F R 1973 The Large Scale Structure of Space-Time (Cambridge: Cambridge University Press)

[20] Javaloyes M A and Sánchez M 2008 A note on the existence of standard splittings for conformally stationary spacetimes Class. Quantum Grav. 25168001

[21] Joshi P S 1993 Global Aspects in Gravitation and Cosmology (Oxford: Clarendon)

[22] Kolassis C A, Santos N O and Tsoubelis D 1988 Energy conditions for an imperfect fluid Class. Quantum Grav. 5 1329-38

[23] Lanczos K 1923 Über eine stationäre Kosmologie im Sinne der Einsteinschen Gravitationstheorie Z. Phys. A $2173-110$

[24] O’Neill B 1983 Semi-Riemannian Geometry with Applications to Relativity (New York, NY: Academic)

[25] Ponge R and Reckziegel H 1993 Twisted products in pseudo Riemannian geometry Geometriae Dedicata 48 15-25

[26] Sánchez M 1999 On the geometry of generalized Robertson-Walker spacetimes: curvature and Killing fields J. Geom. Phys. 31 1-15

[27] Senovilla J M M, Sopuerta C F and Szekeres P 1998 Theorems on shearfree perfect fluids with their Newtonian analogues Gen. Rel. Grav. 30 389-411

[28] van Stockum W J 1937 The gravitational field of a distribution of particles rotating around an axis of symmetry Proc. R. Soc. Edinb. A $\mathbf{5 7} 135$

[29] Tondeur P 1997 Geometry of Foliations (Basel: Birkhäuser)

[30] Wilson F 1970 Some examples of vector fields on the 3-sphere Ann. Inst. Fourier 20 1-20 (http://www.numdam.org/item?id=AIF_1970_20_2_1_0)

[31] Zelmanov A 2006 Chronometric Invariants (Rehoboth, NM: American Research Press) 